\title{
A Comparison of L-Band Helix TWT Experiments with CHRISTINE, a 1-D Multifrequency Helix TWT Code
}

\author{
David K. Abe, Member, IEEE, Mai T. Ngô, Baruch Levush, Senior Member, IEEE, \\ Thomas M. Antonsen, Jr., Member, IEEE, and David P. Chernin
}

\begin{abstract}
Extensive experimental measurements were carried out to test the accuracy of the parametric helix traveling-wave tube (TWT) code, CHRISTINE [1]. The model is one-dimensional, with beam electrons represented as rigid disks. Multifrequency interactions are supported and the RF circuit can be optionally represented with cold-test data, a sheath helix model, or a recently implemented tape helix model [2]. Simulations using the tape helix model are shown to be in good agreement with experimental measurements of an L-band TWT over a broad (250-MHz) frequency range. In the intermediate and saturated power regimes, the modeled and measured TWT gain versus frequency agree to better than $0.4 \mathrm{~dB}$, with deviations explained by strong reflections at the output window that are not accounted for in the code. Single-tone experimental and simulated drive curves agree to better than $1 \mathrm{~dB}$ in the small- and large-signal regimes; relative phase shift simulations agree to within experimental measurement accuracy in the small-signal regime and to within $75 \%$ in the large-signal regime. Two-tone experimental and modeled data exhibit similarly good agreement, with CHRISTINE accurately predicting the effect of frequency-dependent gain variations on the TWT output response and third- and fifth-order intermodulation products.
\end{abstract}

Index Terms-Helix, large-signal one-dimensional (1-D) model, tape helix model, traveling-wave tube.

\section{INTRODUCTION}

$\mathbf{T}$ ELECOMMUNICATIONS, radar, and electronic warfare applications of traveling-wave tube (TWT) amplifiers often require the simultaneous amplification of multiple-frequency signals. However, nonlinearities in the amplifier can lead to the creation of unwanted interaction products, such as amplitude and phase cross modulation and intermodulation. These interaction products reduce the power available to the driven signals and can potentially distort driven signal amplitudes and phases, leading to a degradation in performance, e.g., increased bit-error rates in communications systems. As operating bandwidths and channel densities within bands increase, the problem is exacerbated.

Manuscript received August 30, 1999; revised February 25, 2000. This work was supported by the Office of Naval Research.

D. K. Abe and B. Levush are with the Naval Research Laboratory, Washington, DC 20375 USA (e-mail: david.abe@ nrl.navy.mil).

M. T. Ngô is with the Mission Research Corporation, Newington, VA 22122 USA.

T. M. Antonsen, Jr., is with the Institute for Plasma Research, University of Maryland, College Park, MD 20742 USA, and Science Applications International Corporation, McLean, VA 22102 USA.

D. P. Chernin is with Science Applications International Corporation, McLean, VA 22102 USA.

Publisher Item Identifier S 0093-3813(00)05375-3.
With the projected growth of the telecommunications market and the push toward increased bandwidths to accommodate higher data rates, there is a renewed interest in developing TWT's that can operate at high efficiency with low distortion. Many techniques have been developed to minimize the impact of unwanted distortion. A simple method is to operate the amplifier in its linear regime, backing off from saturation by $6 \mathrm{~dB}$ or more. This method is effective, provided that efficiency is not a key performance criterion. Other more complex techniques have been developed, such as RF predistortion and feedforward compensation [4]. However, these methods have the disadvantage of adding to system complexity, size, and weight, may have bandwidth limitations, and can adversely impact the overall system efficiency.

As an alternative to external compensation schemes, the RF circuit of the TWT itself may be optimized to meet specified criteria. The optimization process can be complex, with tradeoffs made, for example, among output power, linearity, and efficiency. Optimization algorithms have been successfully implemented in coupled-cavity TWT design codes [5]; CHRISTINE was created as a tool to perform similar optimizations of helix TWT circuits under the conditions of multiple input signals and higher order harmonics.

The motivation for the present work is to establish the accuracy of the CHRISTINE model through an extensive comparison of model simulations with experimental measurements of gain, power, phase, amplitude-modulation/phase-modulation (AM/PM) conversion, and intermodulation in a Hughes $8537 \mathrm{H}$ helix TWT. The observed good agreement between the simulated and measured responses establishes the utility of the code as a design tool. With its circuit optimization features, the code can be used to perform complex tradeoff analyzes to produce TWT designs with high efficiency and low distortion. The code has the potential to favorably impact the development time and costs for new TWT designs by reducing the number of hardware iterations required to prove out the designs.

A complete description of the CHRISTINE model can be found in [1]-[3]. Although parametric multifrequency TWT models have been in existence since the mid-1960's [6]-[10], the current code features improved models that produce more accurate simulations of the dispersive behavior of the helix circuit. The earlier models used lossless transmission line approximations of the helix circuit; in the current model, these circuit approximations are replaced by sheath and tape helix models that lead to more accurate calculations of critical 
quantities, such as dispersion and coupling impedance (alternatively, measured cold-test dispersion data can be input from a FORTRAN namelist). Axially dependent RF losses are included in the model.

The sheath helix model has been validated with experimental data taken on a narrowband, $60 \%$ efficient vacuum power booster (VPB) used in a C-band microwave power module (MPM) [11]. Experimental results with a 170-W vacuum power booster (VPB) agreed to within $1 \%$ of simulations. Using this circuit as a starting point, the model was subsequently used to develop an even higher efficiency circuit design; measurements on a prototype TWT built according to this design were in good correlation with the predicted performance [12].

The tape helix model is a recent addition to CHRISTINE [2]. Previous tape helix model implementations have always included some simplifying assumptions about the distribution of surface current densities on the tape, or they have made approximations regarding the manner in which the boundary conditions on the electric field at the tape are enforced. The "narrow tape" approximation seems to be the most common, in which it is assumed that 1) the current flows only along, not across, the tape; 2 ) the current density along the tape is constant across the width of the tape; and 3) $E_{\|}=0$ along the centerline of the tape. The present tape helix model implementation in CHRISTINE makes none of these assumptions; the power flow, interaction impedance, and dispersion for electromagnetic waves propagating on a thin metallic tape helix of arbitrary width are computed exactly, eliminating the need for geometry-correction approximations, such as impedance reduction factors. In the present study, computational models of the $8537 \mathrm{H}$ TWT used the tape helix model exclusively as the most accurate model.

The rest of this paper is organized as follows. Section II describes the mechanical and electrical characteristics of the $8537 \mathrm{H}$ TWT and the implementation of a corresponding CHRISTINE model. Section III compares the results of single-frequency TWT gain, phase, frequency response, and AM/PM conversion measurements with simulations. In Section IV, the results of two-tone TWT experiments are presented and compared with computer simulations and a simple, empirically derived multifrequency model. Overall conclusions are summarized in Section V. Appendix A discusses the sensitivity of the $8537 \mathrm{H}$ to small $( \pm 5 \%)$ variations in the beam voltage, beam radius, and the relative dielectric constant of the helix support rods. The empirical multifrequency model is developed in Appendix B.

\section{Hughes Model 8537H TWT-PhysiCAl DesCRIPTION AND CHRISTINE MODEL}

Effective comparisions between the TWT code and experimental data require detailed information regarding device design and cold- and hot-test performance. Such information is typically unavailable for commercial devices, in which data are either proprietary or not collected in sufficient detail for meaningful model comparisons. In order to generate the necessary data for the present study, a commercial TWT (Hughes model $8537 \mathrm{H}$ ) was found where the manufacturer was willing to provide access to all of the available design and acceptance test

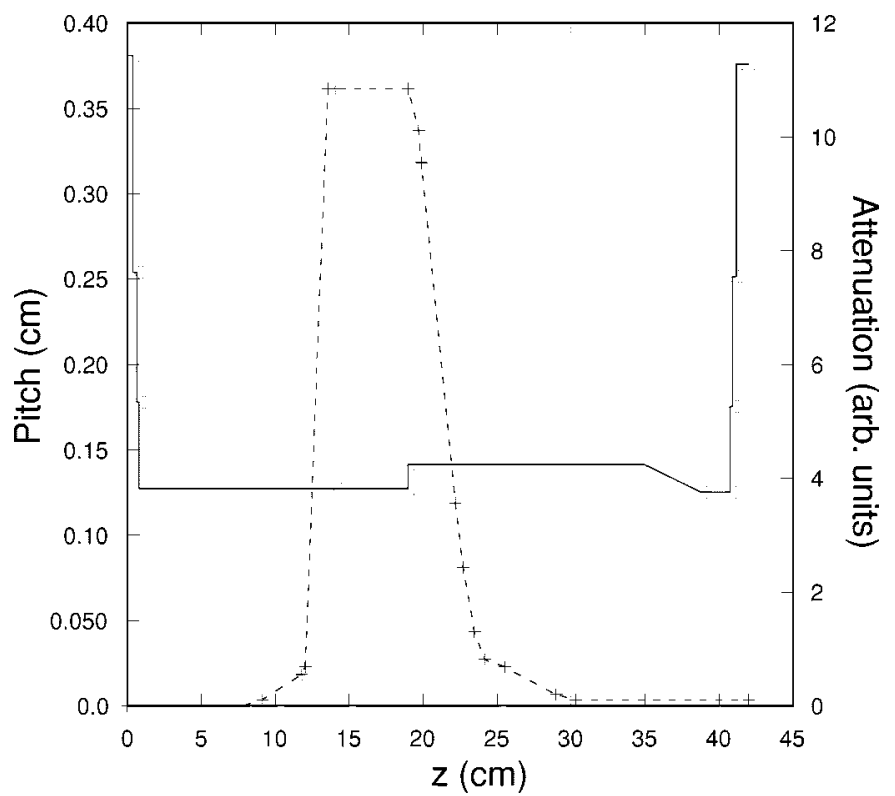

Fig. 1. Helix circuit pitch (solid line) and attenuation (dashed line) as a function of axial position for the Hughes $8537 \mathrm{H}$ TWT.

information; a series of hot-test experiments was subsequently designed and executed. The manufacturer's beam parameter and cold-dispersion data were incomplete, necessitating the normalization of some CHRISTINE model parameters with experimental measurements, as described below.

The $8537 \mathrm{H}$ is a periodic permanent magnet (PPM)-focused, helix TWT designed to produce a nominal $80-\mathrm{W}$ of saturated output power over a frequency range of 1.530 to $1.548 \mathrm{GHz}$. With a guiding magnetic field that is approximately 1.5 times the Brillouin field strength, the electron motion is largely confined to one dimension, making the device a good test vehicle for comparison with one-dimensional (1-D) computer simulations.

Detailed mechanical design information and loss data were made available from the manufacturer. The helix pitch and the circuit attenuation are plotted as a function of axial position in Fig. 1. The slow-wave circuit comprises two constant-pitch helical sections separated by a sever, with a modest circuit taper at the output. As indicated in the figure, three pull-turns are at the input and output of the circuit. The input and output helices are fabricated from circular cross-section tungsten wire that is subsequently ground to a final radial dimension, leaving the wire cross section with a roughly half-moon shape. The circuit is supported within the vacuum envelope by three rectangular cross-section anisotropic pyrolytic boron nitride (APBN) rods. No vanes exist. To control oscillations, the rods are coated with an RF lossy material; the attenuation profile plotted in Fig. 1 is based on empirical measurements of the individual rods. The axial loss and helix dimensions were incorporated into the numerical model without modification; the sever was modeled as an area of very high loss.

The exact electron gun operating voltage and current were not available. The $8537 \mathrm{H}$ is a sealed TWTA system intended for use in space and consists of an electronic power conditioning (EPC) module and TWT, with no accessible cathode diagnostics. Nominal values were available from the manufacturer, but the final optimized test-stand values for a given TWT can vary by as 
TABLE I

HugHeS 8537H-NOMINAL AND MODELED EleCtricAl AND MECHANICAL PARAMETERS

\begin{tabular}{c|c|c}
\hline Parameter & Nominal Value & Model Value \\
\hline Helix mean radius & $0.2353 \mathrm{~cm}$ & $0.2353 \mathrm{~cm}$ \\
Helix wire width & $0.0305 \mathrm{~cm}$ & $0.0305 \mathrm{~cm}$ \\
Cathode voltage & $-3.37 \mathrm{kV}$ & $-3.1 \mathrm{kV}$ \\
Beam current & $70 \mathrm{~mA}$ & $65.5 \mathrm{~mA}$ \\
Min. beam radius & $0.09652 \mathrm{~cm}$ & $0.0962 \mathrm{~cm}$ \\
BN support rods & $1.34($ est'd) & 1.21 \\
$\left(\epsilon_{r}=5.4\right)$ & & \\
\hline
\end{tabular}

much as $\pm 10 \%$ from the nominal design values [13]. To ascertain the gun parameters for the CHRISTINE model used in this study, the mechanical dimensions of the TWT were fixed and the voltage and current were varied within $\pm 10 \%$ until there was agreement with data at selected frequencies. The empirically normalized gun parameters did not vary far from the nominal specifications; as summarized in Table I, the modified voltage and current are within $92 \%$ and $93.6 \%$ of the nominal values, respectively.

The 1-D model approximates the effect that the three APBN support rods have on the dispersive characteristics of the helix circuit by reducing the dielectric permittivity of the rods to an effective "smeared" relative permittivity that completely fills the area between the inner radius of the vacuum envelope and the outer radius of the helix. The value of this smeared relative dielectric constant, $\bar{\epsilon}_{r}$, is computed using area weighting

$$
\bar{\epsilon}_{r}=1+\frac{N_{\text {rod }} A_{\text {rod }}\left(\epsilon_{r}-1\right)}{A_{\text {total }}}
$$

where

$N_{\text {rod }}$ number of rods;

$A_{\text {rod }}$ cross-sectional area of an individual rod;

$\epsilon_{r} \quad$ corresponding rod relative dielectric constant;

$A_{\text {total }}$ total cross-sectional area between the vacuum envelope and the outer surface of the helix.

The nominal relative permittivity of the APBN rods is $\epsilon_{r}=$ 5.4. From (1), the calculated equivalent relative permittivity is $\bar{\epsilon}_{r}=1.34$. However, comparison with measured phase data indicates that $\bar{\epsilon}_{r}=1.21$ yields better agreement with data. The principle reason for this discrepancy is the inaccuracy of the 1-D approximation and the implicit assumption of a constant radial electric field between the helix and the vacuum envelope wall. Although (1) can yield a good first-order approximation, realistically, the effective relative permittivity must be treated as a "fit" parameter to fine-tune the TWT model.

The nominal and "as-modeled" mechanical and electrical parameters for the $8537 \mathrm{H}$ TWT are summarized in Table I. The sensitivity of the predicted gain and phase to the beam voltage, minimum radius, and $\bar{\epsilon}_{r}$ is examined in Appendix A.

Both single-frequency and multifrequency simulations included the effect of second harmonic interaction. In single-frequency tests, the inclusion of second harmonics has been shown to markedly improve the agreement of the model with experimental data [11]. Similar accuracy enhancements

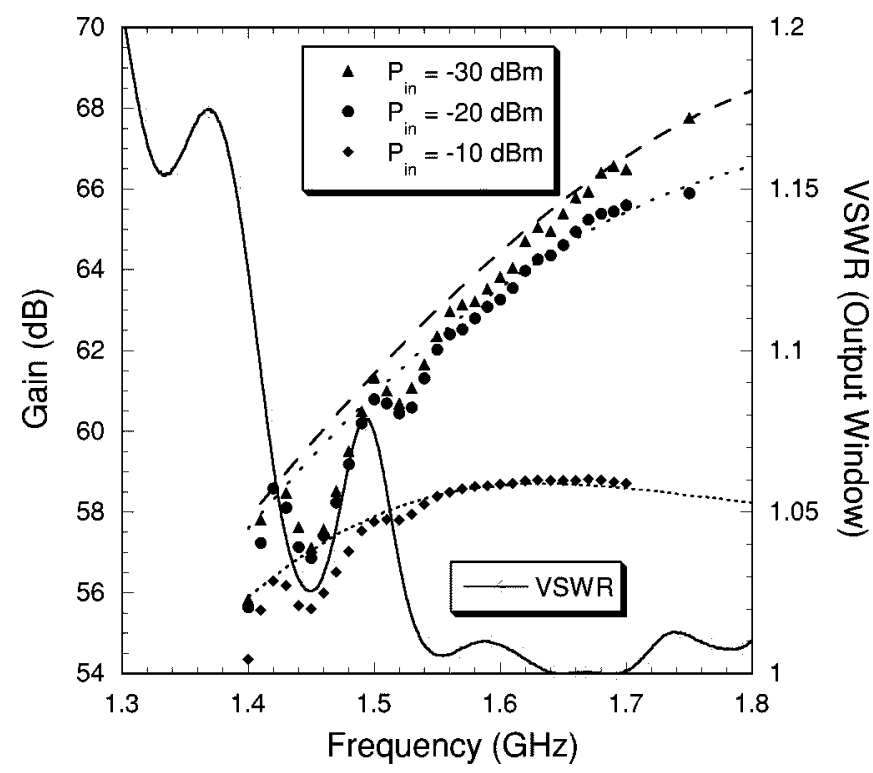

Fig. 2. Simulated and experimental gain versus frequency for various input powers with the output window VSWR superimposed (CHRISTINE predictions are plotted as dashed/dotted lines).

resulting from the inclusion of second harmonics were observed in the present study.

The simulated interaction region of the $8537 \mathrm{H}$ was broken into 600 axial zones. The beam was assumed to be cold, and the number of entrance phases was typically set at 137 . To reach convergence, single-frequency simulations required 10 space charge harmonics and multifrequency simulations required 15 space charge harmonics. Typically, run times ranged from less than a minute for single-frequency runs to several hours for multifrequency runs.

\section{SingLE-FrequenCy EXPERIMENTS AND SimUlations}

In this section, the results of CHRISTINE simulations are compared with experimental measurements of TWT performance under single-tone excitation.

\section{A. Frequency Dependence}

The usable amplification band of the 8537H TWT far exceeds the $18-\mathrm{MHz}(1.53 \mathrm{GHz} \leq f \leq 1.548 \mathrm{GHz})$ civil communications frequency band for which the device was designed. To better study the capabilities of the code, experimental measurements were made over a 350-MHz wide band, ranging from 1.40 to $1.75 \mathrm{GHz}$.

Fig. 2 compares gain versus frequency simulations using the TWT model of Table I with experimental measurements taken at three different drive levels: $P_{\text {in }}=-10 \mathrm{dBm}$ (corresponding to a point near the saturated output of the TWT); $P_{\mathrm{in}}=-30 \mathrm{dBm}$ (in the small-signal regime); and $P_{\text {in }}=-20 \mathrm{dBm}$ (in an intermediate regime). The VSWR of the $8537 \mathrm{H}$ output window as a function of frequency is superimposed on the gain plots of Fig. 2. As can be seen in the figure, the output window begins to exhibit strong reflections below a frequency of $\sim 1.52 \mathrm{GHz}$. Correspondingly, the agreement between the model and experiment falls off below $1.52 \mathrm{GHz}$, as CHRISTINE does not account for reflections 


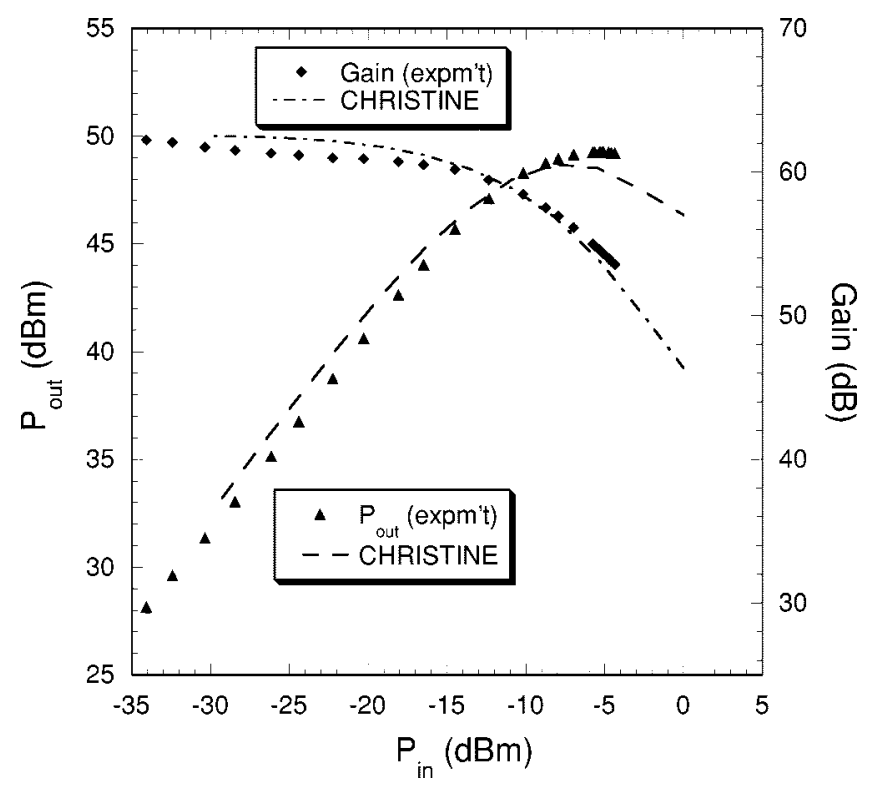

(a)

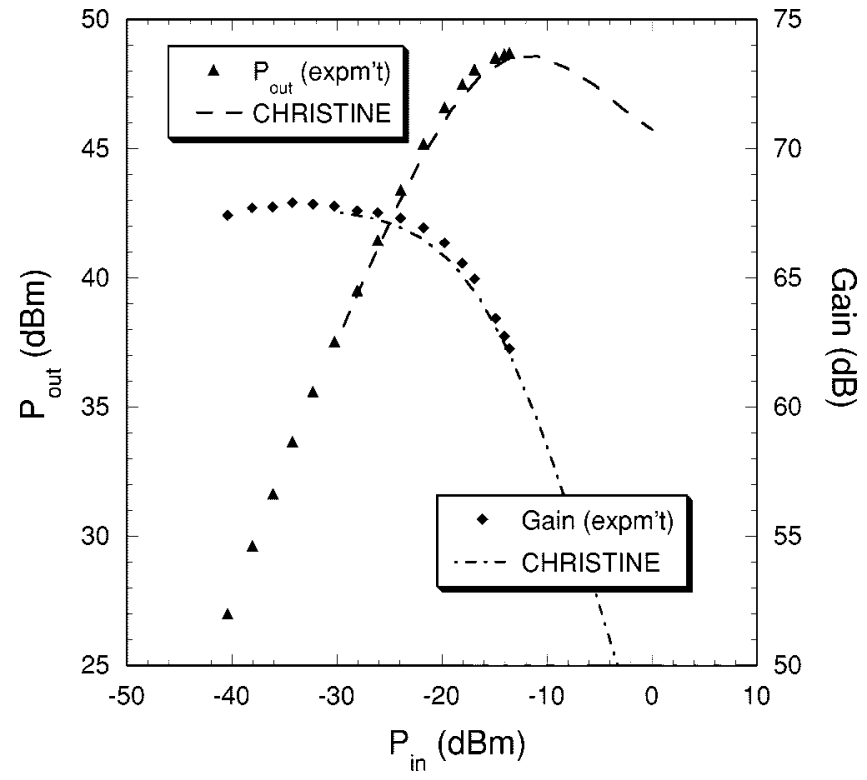

(b)

Fig. 3. Simulated and experimental drive and gain curves for (a) $f=1.538$ $\mathrm{GHz}$ and (b) $f=1.75 \mathrm{GHz}$.

from the output window. Above $1.52 \mathrm{GHz}$, the VSWR drops below 1.02 and the agreement improves markedly. In the small-signal regime $\left(P_{\text {in }}=-30 \mathrm{dBm}\right)$, model simulations and experimental measurements above $1.54 \mathrm{GHz}$ agree to better than $84 \%$. In the intermediate and near-saturation regimes, predicted and measured TWT gain agree to better than $92 \%$ and $98 \%$, respectively.

\section{B. Drive and Gain Curves}

TWT gain and output power are plotted as a function of input power in Fig. 3 for two extremes in frequency: a) $f=1.538$ $\mathrm{GHz}$ and b) $f=1.75 \mathrm{GHz}$. The agreement between the CHRISTINE simulations and the measured data is very good, with discrepancies of $\leq 1 \mathrm{~dB}$ in both the small- and large-signal regimes.

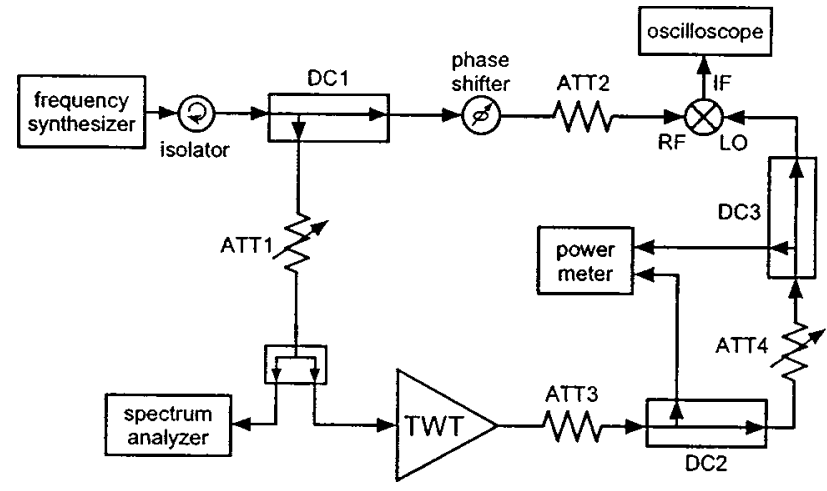

Fig. 4. Experimental layout for phase shift measurements.

The quality of agreement seen in Fig. 3 is typical of the agreement seen across the entire $250-\mathrm{MHz}$ passband under study.

\section{Relative Phase Shift and AM/PM Conversion}

Fig. 4 is a simplified block diagram of the experimental setup used to measure the phase shift through the $8537 \mathrm{H}$ TWT as a function of input power (at fixed frequencies). A double-balanced mixer with a DC-coupled IF port was used as the phase detector. At a given frequency, the phase delays in the measurement system are the result of constant delays caused by fixed attenuators and cabling, phase delays dependent on variable attenuator dial settings, and the phase delay introduced by the TWT. The constant phase delays in the measurement system were cancelled out by referencing the measured phase shifts as a function of drive power to a constant small-signal phase. The phase delays through the phase shifter and the variable attenuators were calibrated as a function of the dial setting at a number of frequencies using a vector network analyzer.

To measure the relative phase shift through the TWT, a fixedamplitude, synthesized RF input signal was split at directional coupler DC1, with part of the signal applied to the RF port of the double-balanced mixer through a phase shifter and fixed attenuator (ATT2) and part applied to the input of the TWT through a variable attenuator (ATT1). As the input power to the TWT was swept using variable attentuator ATT1, the power at the LO port of the mixer was held constant using variable attenuator ATT4. In the limit of small-signal input drive, the phase delay through a TWT is a constant. For the $8537 \mathrm{H}$, this input power was typically $P_{\text {in }} \leq-30 \mathrm{dBm}$. Using $P_{\text {in }} \simeq-30 \mathrm{dBm}$, an initial phase reference point was established using the phase shifter to place the LO and RF ports of the mixer in quadrature. As the input drive power to the TWT was increased, the phase delay through the TWT increased and the phase at the LO port (relative to the initial small-signal phase) had to be adjusted with the phase shifter to maintain quadrature. When combined with the phase calibrations of the phase shifter and variable attenuators, the phase difference read on the phase shifter is a measure of the phase shift relative to the small-signal phase constant in the TWT.

Fig. 5 is a plot of the simulated and measured relative phase shift through the TWT as a function of drive power at a frequency of $1.56 \mathrm{GHz}$. The predicted phase shift exhibits the same 


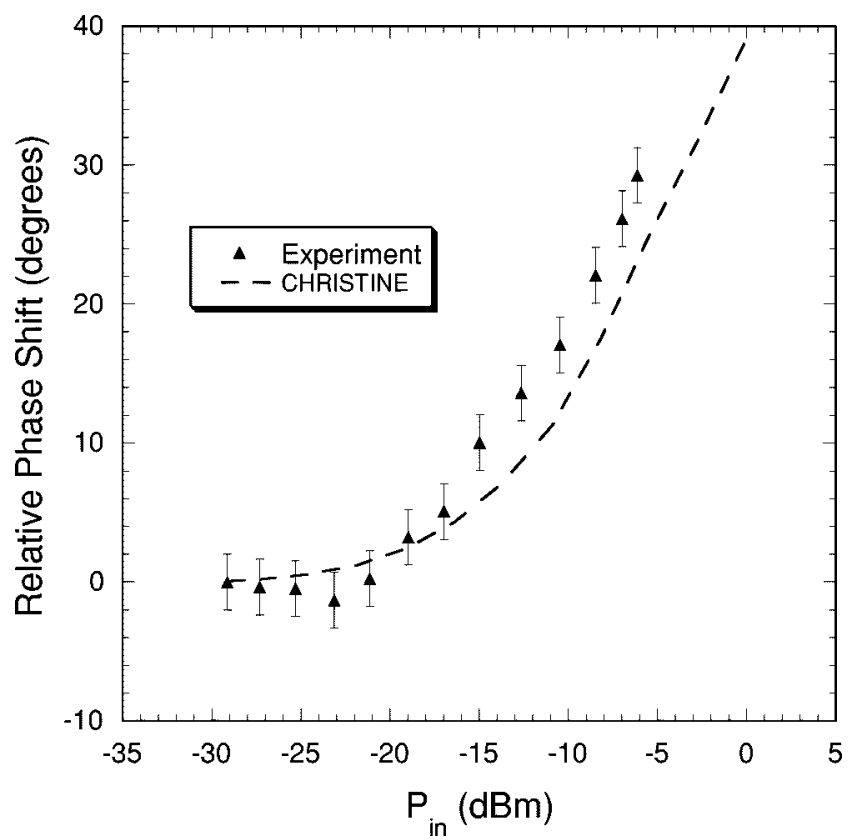

Fig. 5. Simulated and experimental phase shift versus drive power curves for $f=1.56 \mathrm{GHz}$.

trends as the measured phase shift, beginning with a constant phase in the small-signal regime of $P_{\text {in }} \leq-30 \mathrm{dBm}$, and increasing to a phase shift of approximately $25^{\circ}$ at saturation $\left(P_{\mathrm{in}} \simeq-6 \mathrm{dBm}\right)$; in comparison, the measured phase shift at saturation was approximately $29^{\circ}$. The accuracy of the experimentally measured phase (indicated by the $\pm 2^{\circ}$ of error bars on the experimental data) is limited by the reproducibility of the dial settings on the variable attenuators used in the measurement setup of Fig. 4. The remaining discrepancy between the measured data and the simulations is most likely from factors affecting the CHRISTINE computation of dispersion in the TWT, including the limitations of a 1-D model and inhomogeneities in the permittivity of the APBN support rods.

$\mathrm{AM} / \mathrm{PM}$ conversion is a measure of the change in phase between the input and output of an amplifier as a function of drive power and is computed as the derivative the phase versus drive power curve. Physically, as the drive level is increased, the average electron beam velocity decreases as the particles interact with the applied electromagnetic wave and give up kinetic energy to the propagating wave; subsequently, the phase delay and $\mathrm{AM} / \mathrm{PM}$ conversion increases.

Fig. 6 is a plot of the simulated and experimentally derived $\mathrm{AM} / \mathrm{PM}$ conversion as a function of input power relative to saturation for the $8537 \mathrm{H}$ TWT driven at a frequency of $1.56 \mathrm{GHz}$. The measured and predicted AM/PM response shown in the figure is typical for a TWT: the AM/PM conversion is neglible in the small-signal regime, slowly increases as the drive power is increased, and finally, sharply increases as the device nears saturation. To obtain the experimental AM/PM curve of Fig. 6, a cubic spline was fit to the relative phase shift data of Fig. 5 and the first derivative of this fit was plotted as a function of the drive power relative to saturation. The analysis is sensitive to small fluctuations in the original phase data; the structure seen in the experimental AM/PM curve is in part caused by uncertainties in the experimental phase measurement.

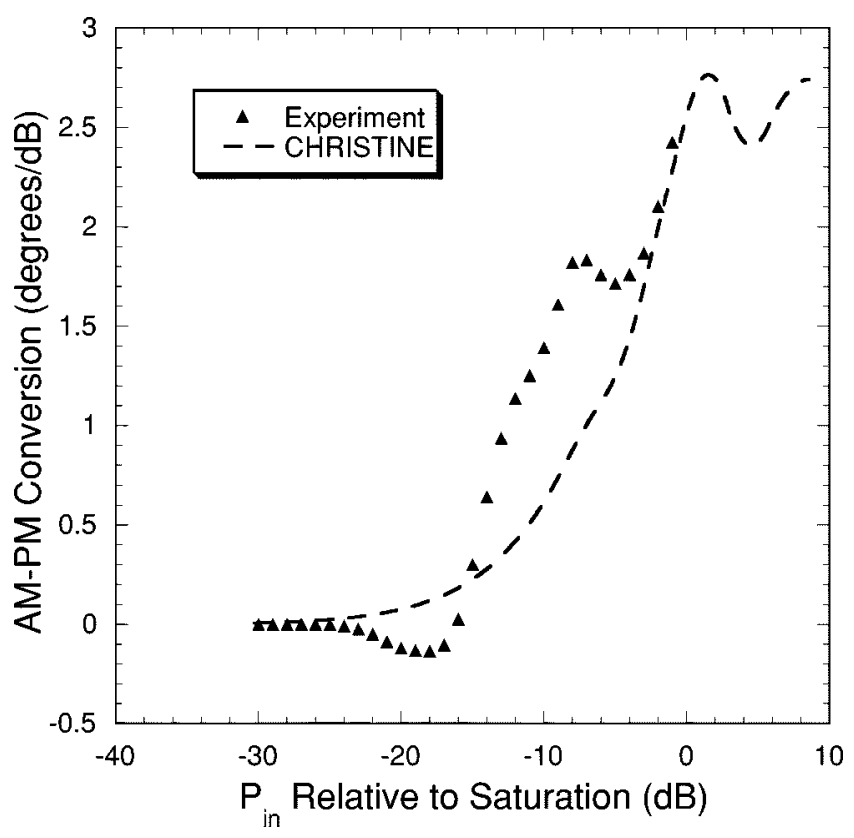

Fig. 6. Simulated and experimental AM/PM conversion versus drive power relative to saturation for $f=1.56 \mathrm{GHz}$.

\section{MultifReQuenCy EXPERIMENTS AND SimUlations}

In this section, CHRISTINE simulations of intermodulation distortion are compared with experimental data. In addition, results from a simple multifrequency model based on a nonlinear fit to an experimental single-carrier gain-frequency curve are compared with CHRISTINE and experiment.

\section{A. CHRISTINE Two-Tone Simulations}

To evaluate the multifrequency performance of the code, a series of two-tone experiments was carried out with the $8537 \mathrm{H}$ TWT. In the experiments, two signals of equal power amplitudes and dissimilar frequencies were combined in a hybrid junction and applied to the input of the TWT. One tone was kept at a constant frequency of $f_{0}=1.54 \mathrm{GHz}$, whereas the other tone varied in frequency from $1 \mathrm{MHz}$ up to $50 \mathrm{MHz}$ from $f_{0}$. The output power as a function of the input drive power of the amplified tones and the resulting intermodulation products were measured with a spectrum analyzer.

The TWT model used in the intermodulation studies was identical to the basic TWT model developed in the single-frequency studies (cf. Fig. 1 and Table I). As in the intermodulation experiments, two equal-amplitude driven tones were input into the model and a sufficient number of $\omega_{n}$ frequency components were defined to span a fundamental frequency range that encompassed the driven tones plus the third- and fifth-order intermodulation products.

Fig. 7 is a plot of the experimentally measured output of the TWT (amplified driven tones, third- and fifth-order intermodulation products) along with CHRISTINE model simulations for the case of two equal-amplitude driven tones separated by $30 \mathrm{MHz}$. For the amplified driven tones, the agreement between the model and the data is quite good, with less than $1 \mathrm{~dB}$ of discrepancy between simulation and experiment. In the 


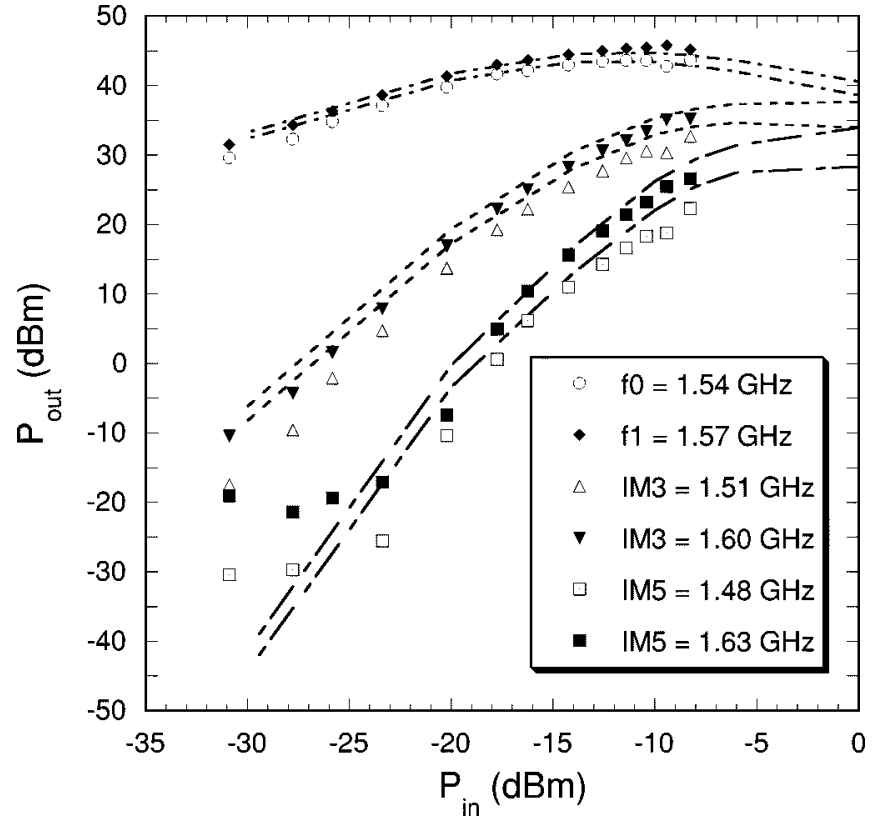

Fig. 7. Experimental driven tones $\left(f_{0}=1.54 \mathrm{GHz}, f_{1}=1.57 \mathrm{GHz}\right)$ and third-(IM3) and fifth-(IM5) order intermodulation products (CHRISTINE simulations shown as dashed lines).

intermediate-to-near-saturation regime, the predicted and measured third- and fifth-order intermodulation responses are also in good agreement, with discrepancies of less than $1.5 \mathrm{~dB}$. In the small-signal regime, $P_{\text {in }} \leq-20 \mathrm{dBm}$, there is an anomalous nonlinearity in the measured intermodulation waveforms, where the third- and fifth-order responses approach asymptotic values for decreasing input power amplitudes instead of linearly decreasing, as indicated by the code. The experimental measurement system was rigorously calibrated; this behavior was also observed by an independent researcher using a different measurement setup [14]; it is unlikely that this effect is an artifact of the measurement.

\section{B. Comparison of a Simple Multifrequency Model with Experimental Measurements and CHRISTINE}

The single-carrier amplitude response versus input power and phase response versus input power are two of the most fundamental characteristics of a nonlinear amplifier. Because of the relative simplicity of these measurements, it is attractive to attempt to develop a multifrequency TWT theory based the measured amplifier response at a only a single frequency [15], [16]. A simple multifrequency model based on an expansion about a single-frequency complex gain curve is developed in detail in Appendix B. In this section, the predictions made by this model are compared to experiment and CHRISTINE simulations. Not unexpectedly, the simple multifrequency model is seen to be most accurate when the average carrier frequency is close to the single-carrier (fit) frequency and the frequency separation between carriers is small; the CHRISTINE model is more accurate for arbitrary frequencies and greater carrier separations.

Following the development of Appendix B, a complex gain function of the form described by (B.3a) was created by fitting a fourth-order polynomial to the measured single-frequency gain and phase response $\left(f_{0}=1.54 \mathrm{GHz}\right)$ of the Hughes $8537 \mathrm{H}$
TWT. The output response (amplified driven tones, and thirdand fifth-order intermodulation products) was computed from (B.6) and (B.7) and compared with measured data and CHRISTINE simulations.

Fig. 8 compares the predictions of the simple multifrequency model with the measured output response of the TWT for the case of two equal-amplitude input tones separated by $\Delta f=1 \mathrm{MHz}$ in frequency $\left(f_{0}=1.54 \mathrm{GHz}, f_{1}=1.539 \mathrm{GHz}\right)$. The amplified driven tone response, third-order intermodulation products, and fifth-order intermodulation products versus RF drive power are plotted in Fig. 8(a)-(c), respectively. With a frequency separation of only $1 \mathrm{MHz}$, the gain of the TWT is nearly constant and the output responses resulting from the two input frequencies are identical to within experimental accuracy. For this case, the assumptions regarding gain invariance with bandwidth inherent in (B.2) are valid, and unsurprisingly, the simple model simulation-based on a fit to single frequency complex gain data at $f_{0}=1.54 \mathrm{GHz}$ - is in excellent agreement with the experiment, as seen in the dotted line plots of Fig. 8. The agreement between the CHRISTINE simulations and the measured response is also good, with maximum discrepancies between the model and the data occurring in the small-signal regime. These discrepancies are on the order of $1 \mathrm{~dB}$ for the amplified driven tones and up to $5 \mathrm{~dB}$ for the third- and fifth-order intermodulation products. As was seen in Fig. 7, there is a nonlinearity in the empirical small-signal intermodulation response that is not accounted for by either model.

The TWT output response from a larger two-tone separation of $\Delta f=50 \mathrm{MHz}\left(f_{0}=1.54 \mathrm{GHz}, f_{1}=1.59 \mathrm{GHz}\right)$ is plotted in Fig. 9. In this case, as seen in Fig. 2, the TWT gain is not constant over the driven bandwidth but instead varies by several decibels in the small-signal and intermediate-signal regimes. As a result, the amplified responses of the driven tones and the resulting pairs of third- and fifth-order intermodulation products have distinct power amplitudes. For example, referring to Fig. 9(a), the two experimental amplified drive curves (indicated by symbols) corresponding to the drive frequencies of 1.54 and $1.59 \mathrm{GHz}$ can be seen to differ by 2 to $4 \mathrm{dBm}$ in output power over the range of swept drive power. Under these conditions, the simple multifrequency model of Appendix B, which assumed that the gain was independent of frequency, breaks down. Although the simple model does follow the $1.54 \mathrm{GHz}$ and lower frequency branch intermodulation data, it fails to predict the two distinct, frequency-dependent output responses and overpredicts the saturated responses.

In contrast, the multifrequency model of CHRISTINE predicts the distinct, frequency-dependent output responses. The accuracy of the simulations is best near saturation, where the code and the measured data agree to within $1 \mathrm{~dB}$ for the amplified driven tones and to within 2 to $3 \mathrm{~dB}$ for the intermodulation products. In the intermediate/small-signal regime, the agreement is within $3 \mathrm{~dB}$ for the amplified driven tones and intermodulation products.

\section{CONCLUSION}

Simulation results from the 1-D, parametric, helix TWT code, CHRISTINE, have been compared with the experiment. Using a newly implemented tape helix model, the code has 


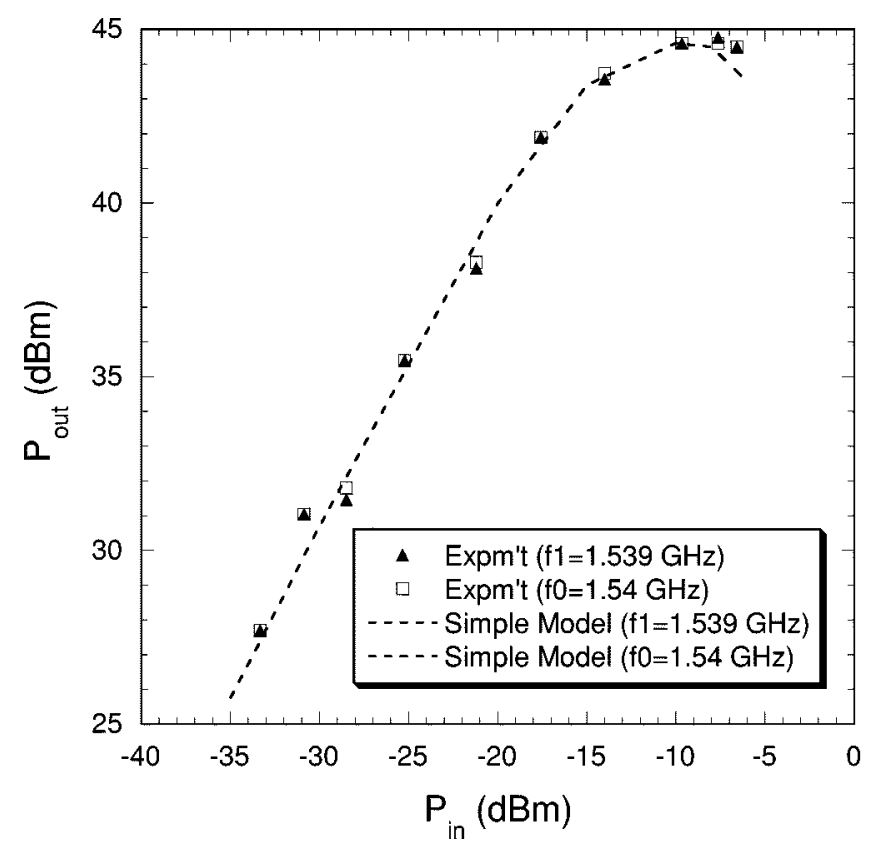

(a)

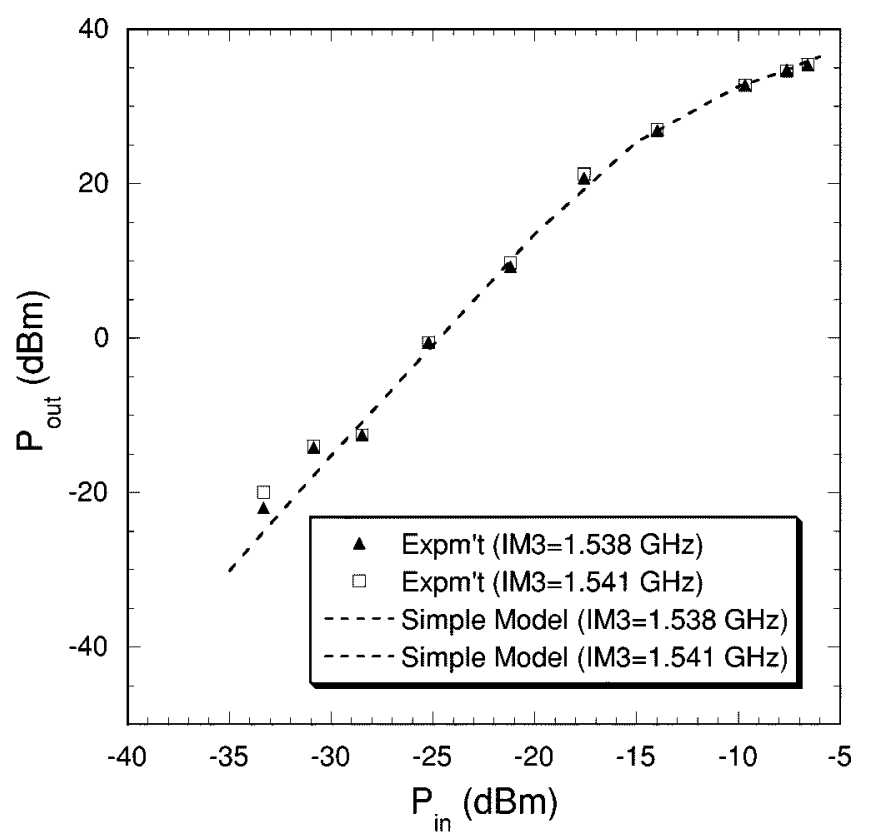

(b)

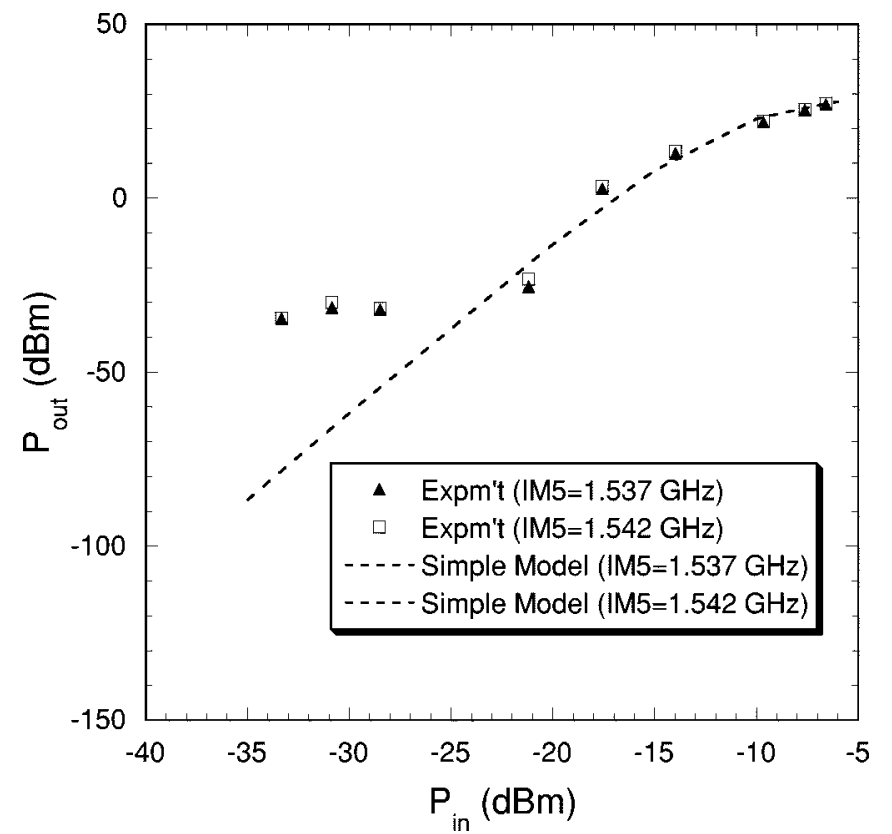

(c)

Fig. 8. Comparison of the simple multifrequency model of Section $\mathrm{V}$ with experimental intermodulation measurements for driven tone frequencies of $f_{0}=1.54$ GHz and $f_{1}=1.539 \mathrm{GHz}$ : (a) amplified driven tones; (b) third-order intermodulation products; and (c) fifth-order intermodulation products.

been shown to accurately simulate the performance of a Hughes Model 8537H L-band TWT, with both single- and multiple-tone excitation. Over a $250-\mathrm{MHz}$ evaluation passband, predicted single-frequency drive and phase curves agreed to within $1 \mathrm{~dB}$ of experimental measurements; multifrequency simulations using two tones separated by as much as $50 \mathrm{MHz}$ were in similar good agreement to experimental data, including thirdand fifth-order intermodulation products. With its demonstrated accuracy and efficient run-times, CHRISTINE is a valuable tool for the future development of high-performance helix TWT's. With its flexible optimization and multifrequency capabilities, the code is particularly useful in design applications in which high efficiency and low distortion products are essential, such as ground- and space-based telecommunications and electronic countermeasures.

\section{APPENDIX A}

\section{SENSITIVITY OF THE 8537H NUMERICAL MODEL TO SMALL} Changes in Key Parameters

The sensitivity of the $8537 \mathrm{H}$ TWT to small $( \pm 5 \%)$ changes in the cathode voltage, beam radius, and effective dielectric constant was studied with the CHRISTINE numerical model using the parameters described by Fig. 1 and Table I. The effects of 


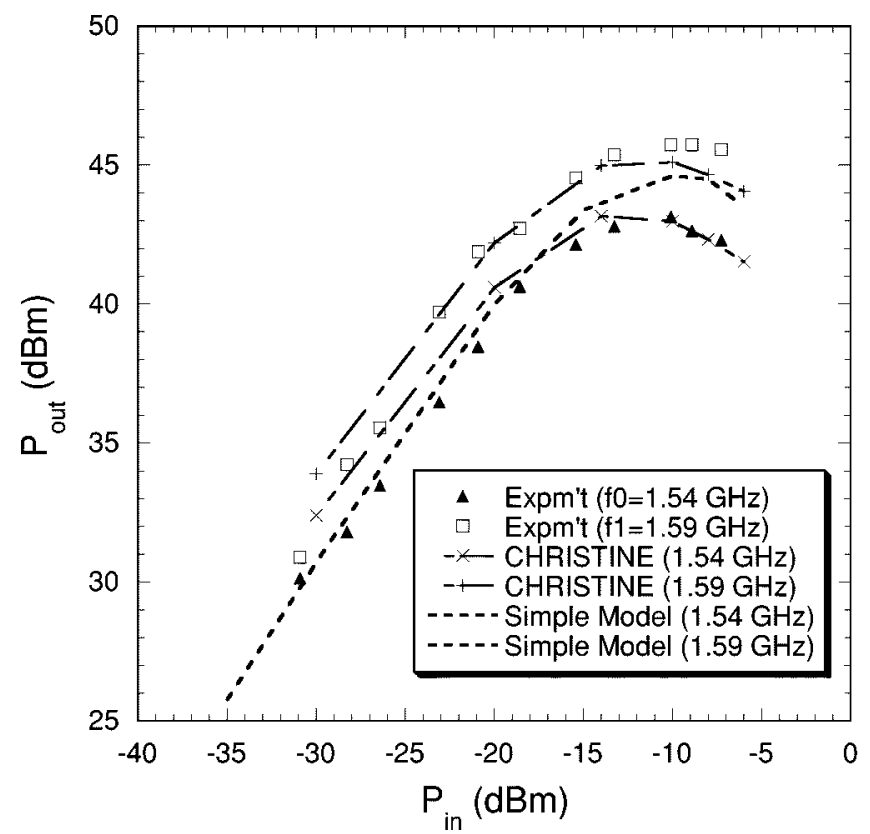

(a)

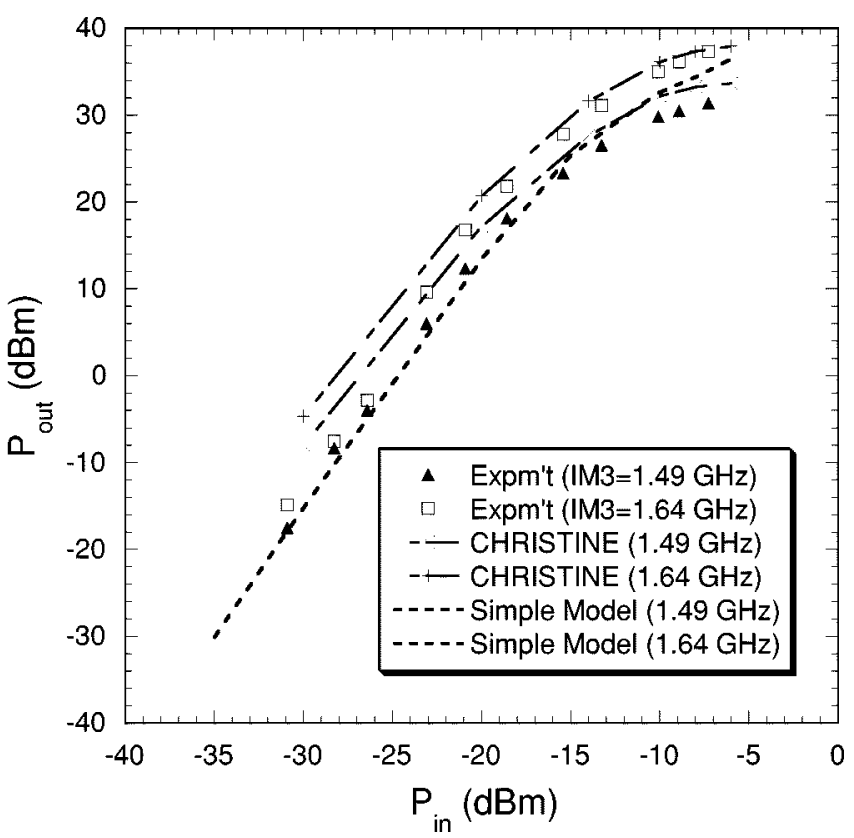

(b)

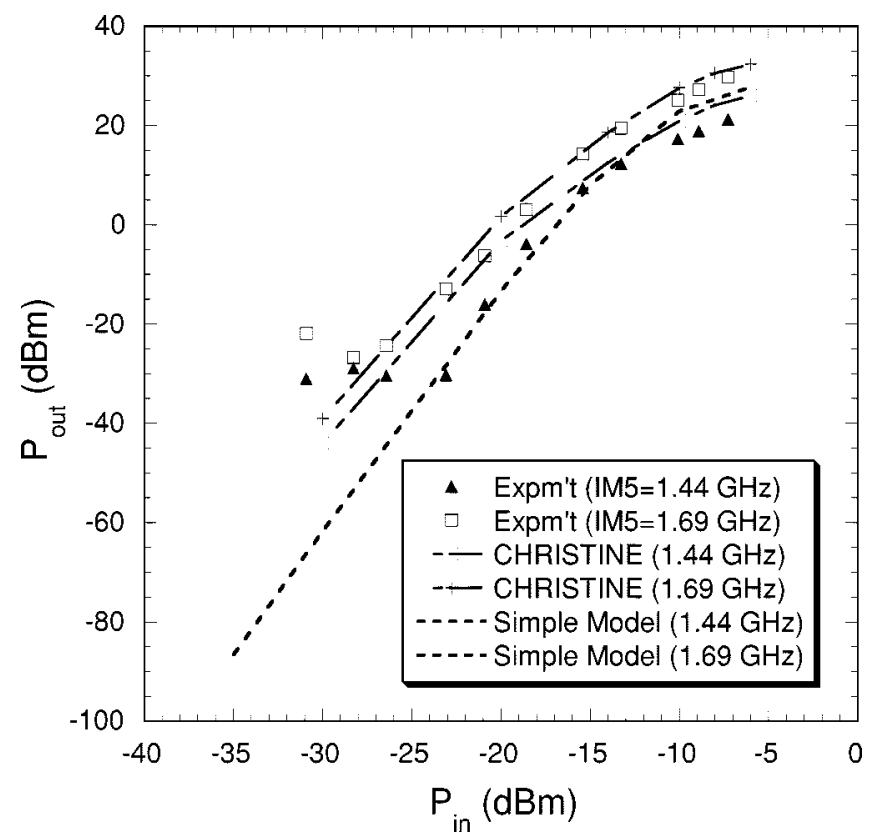

(c)

Fig. 9. Comparison of experimental intermodulation measurements with predictions by CHRISTINE and the simple multifrequency model of Section V for driven tone frequencies of $f_{0}=1.54 \mathrm{GHz}$ and $f_{1}=1.59 \mathrm{GHz}$ : (a) amplified driven tones; (b) third-order intermodulation products; and (c) fifth-order intermodulation products.

these variations on the small- and large-signal gain and relative phase shift responses are summarized in Figs. 10-12.

Figs. 10 and 11 are plots of the simulated $8537 \mathrm{H}$ gain versus frequency and the relative phase shift versus input power for $\pm 5 \%$ variations in beam voltage and radius. Both small-signal $\left(P_{\text {in }}=-30 \mathrm{dBm}\right)$ and large-signal $\left(P_{\text {in }}=-10 \mathrm{dBm}\right)$ responses are plotted. As expected, the relative phase shift and the small-signal gain were sensitive to the input parameters, whereas the large-signal gain was relatively insensitive.

Small-signal analysis can help to develop some physical insight on the effect that variations in voltage and radius can have on TWT performance. From [17], the small-signal gain is pro- portional to the product of the Pierce gain parameter $C$ and length of the helix in wavelengths

$$
C N=\left(\frac{Z_{c} I_{b}}{4 V_{b}}\right)^{1 / 3}\left(\frac{\omega L}{2 \pi u_{0}}\right)
$$

where

$\omega$ radian frequency;

$L$ helix length;

$u_{0} \quad \sqrt{2 \eta_{e} V_{b}}$;

$\eta_{e} \quad$ electron charge-to-mass ratio $\left(e / m_{e}\right)$;

$Z_{c}$ interaction impedance. 


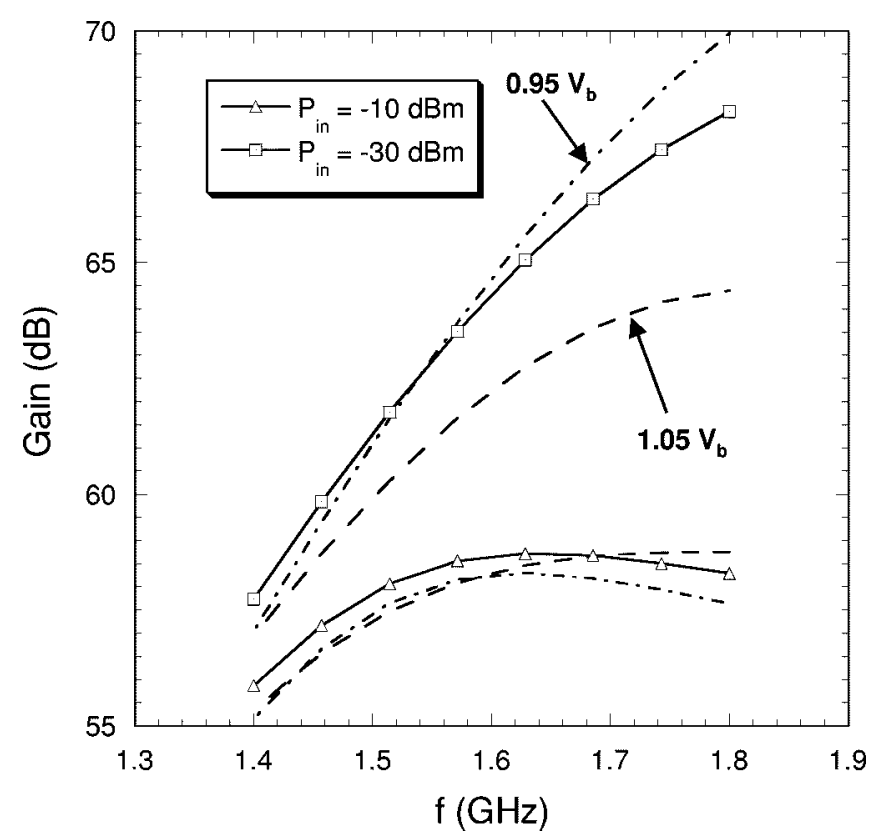

(a)

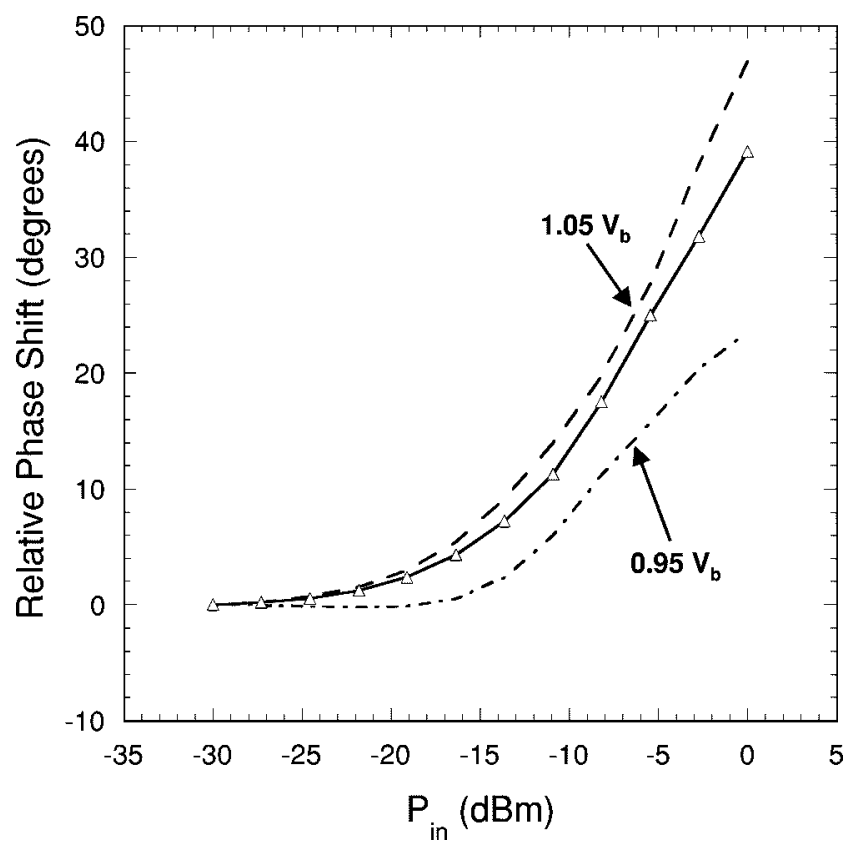

(b)

Fig. 10. The effect of $\pm 5 \%$ variations in beam voltage on (a) gain versus frequency and (b) relative phase shift versus input power (at $f=1.56 \mathrm{GHz}$ ) in the small-signal $\left(P_{\text {in }}=-30 \mathrm{dBm}\right)$ and near-saturation $\left(P_{\text {in }}=-10 \mathrm{dBm}\right)$ regimes. The solid lines are the responses for the nominal modeling parameters of Table I, and the dot-dash and dashed lines represent $0.95 \mathrm{~V}_{b}$ and $1.05 \mathrm{~V}_{b}$, respectively.

With a cathode potential of $V_{b}=3100 \mathrm{~V}$, the electron velocity in the $8537 \mathrm{H}$ TWT is much smaller than is the speed of light $\left(u_{0} \simeq 0.11 c\right)$, and the interaction impedance $Z_{c}$ can be approximated by

$$
Z_{c}=\frac{\left|E_{z}\right|^{2}}{2 \beta^{2} P} \simeq \frac{c}{2 u_{0}}\left[R\left(\gamma r_{b}\right) F(\gamma a)\right]^{3}
$$

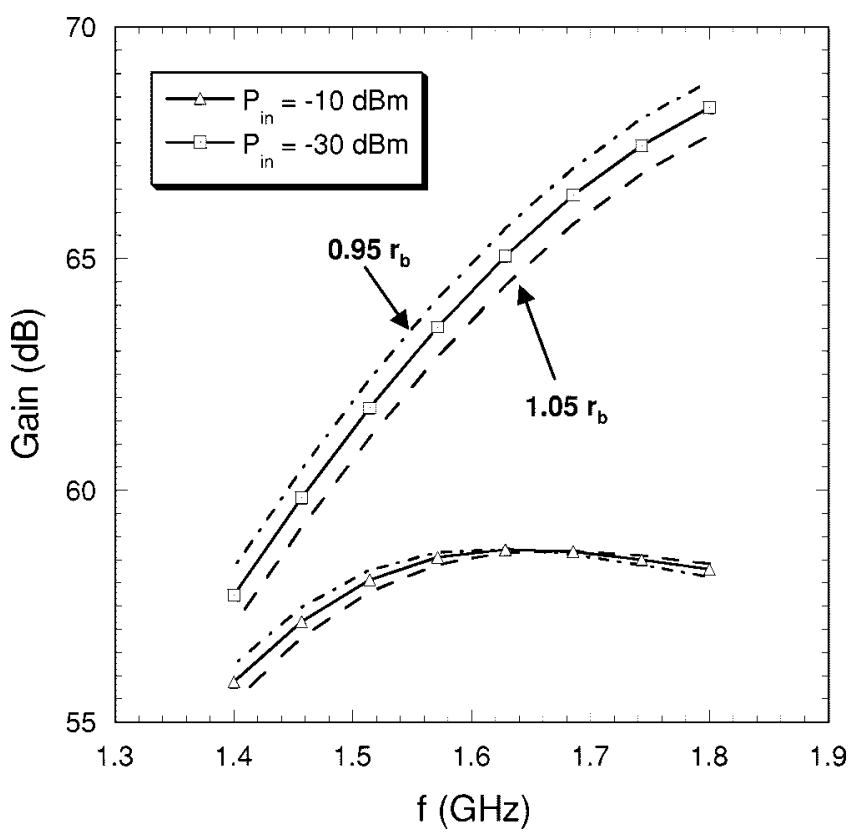

(a)

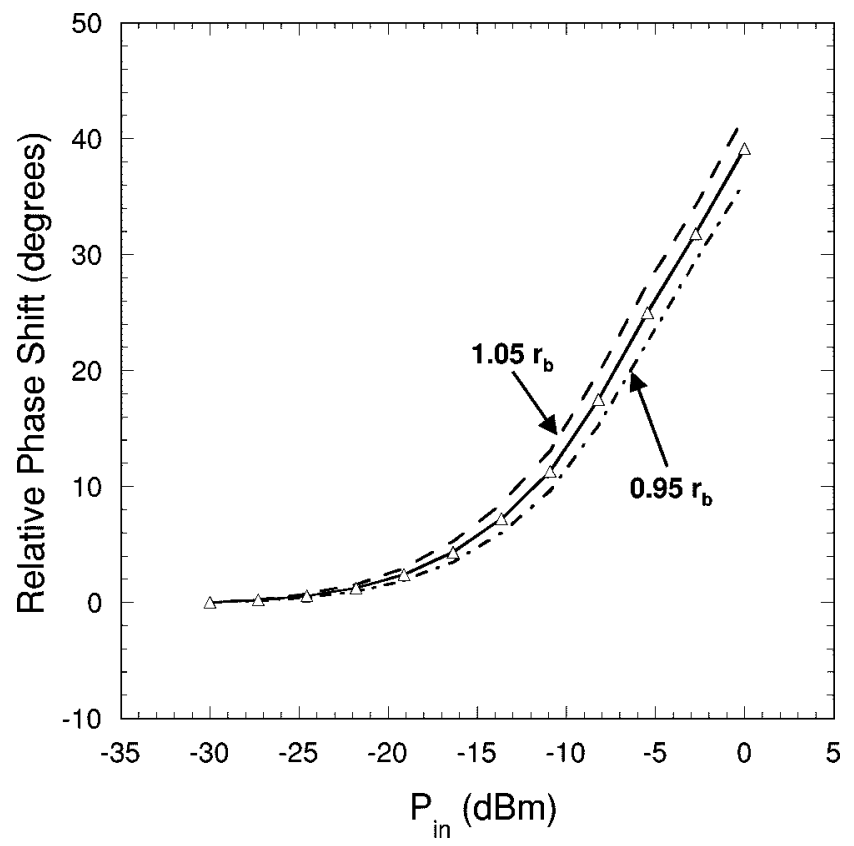

(b)

Fig. 11. The effect of $\pm 5 \%$ variations in beam radius on (a) gain versus frequency and (b) relative phase shift versus input power (at $f=1.56 \mathrm{GHz}$ ) in the small-signal $\left(P_{\mathrm{in}}=-30 \mathrm{dBm}\right)$ and near-saturation $\left(P_{\mathrm{in}}=-10 \mathrm{dBm}\right)$ regimes. The solid lines are the responses for the nominal modeling parameters of Table I, and the dot-dash and dashed lines represent $0.95 r_{b}$ and $1.05 r_{b}$, respectively.

where $a$ is the helix radius, $\gamma=\sqrt{\beta^{2}-\beta_{0}^{2}}, \beta=\omega / v_{\mathrm{ph}}, \beta_{0}=$ $\omega / c$, and we have assumed synchronism between the beam and the wave on the helix so that $v_{\mathrm{ph}} \sim u_{0}$. The impedance function $F$ is proportional to the interaction impedance on the axis of the helix. The interaction impedance at the beam radius $r_{b}$ requires $F$ to be modified by the impedance reduction factor $R\left(\gamma r_{b}\right)$. For solid beams, $R$ is given by [17]

$$
R\left(\gamma r_{b}\right)=\left[I_{0}^{2}\left(\gamma r_{b}\right)-I_{1}\left(\gamma r_{b}\right)\right]^{1 / 3} .
$$




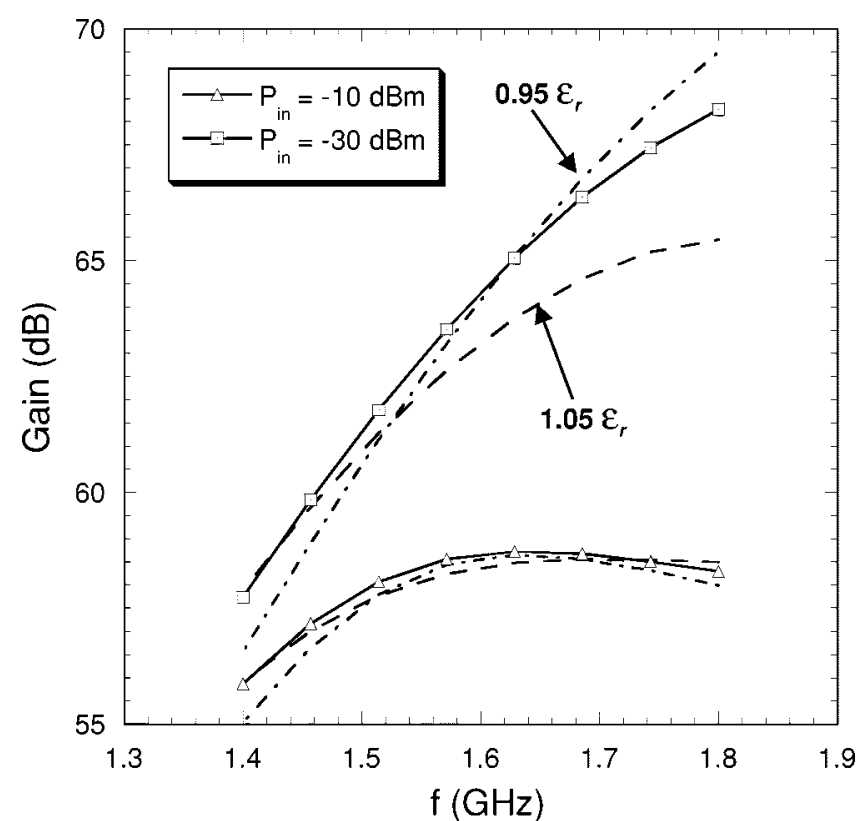

(a)

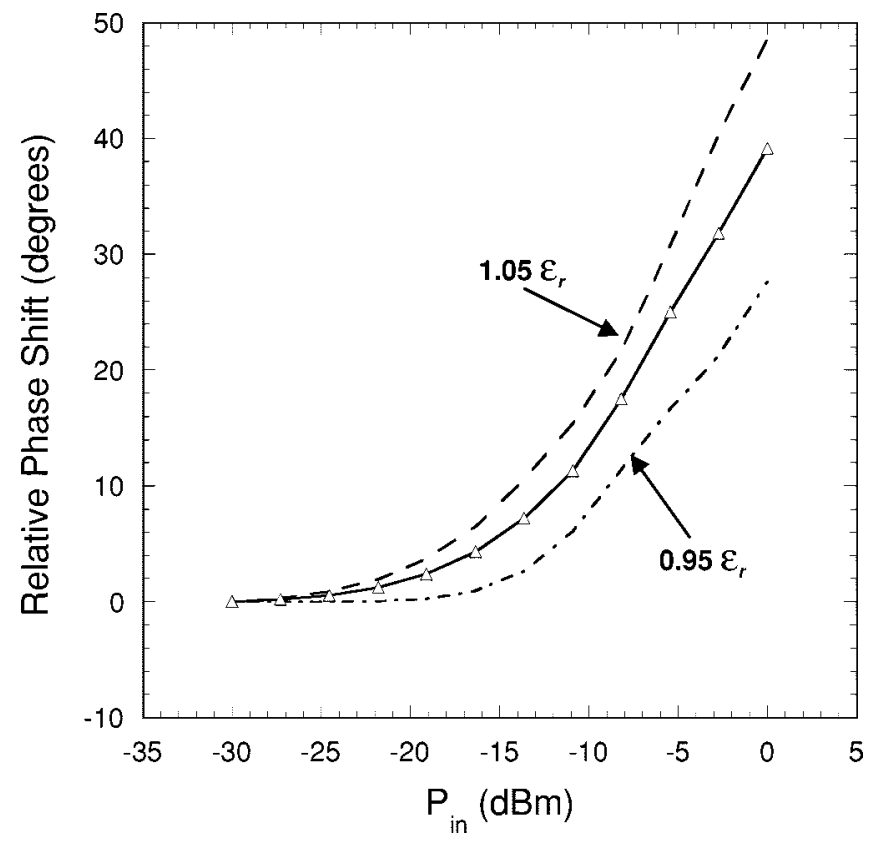

(b)

Fig. 12. The effect of $\pm 5 \%$ variations in the effective relative dielectric constant $\bar{\epsilon}_{r}$ on (a) gain versus frequency and (b) relative phase shift versus input power (at $f=1.56 \mathrm{GHz})$ in the small-signal $\left(P_{\mathrm{in}}=-30 \mathrm{dBm}\right)$ and near-saturation $\left(P_{\mathrm{in}}=-10 \mathrm{dBm}\right)$ regimes. The solid lines are the responses for the nominal modeling parameters of Table I, and the dot-dash and dashed lines represent $0.95 r_{b}$ and $1.05 r_{b}$, respectively.

In the case of the $8537 \mathrm{H}, R \sim 1$ over a $\pm 5 \%$ variation in beam radius. Combining (A.1) and (A.2), the proportional gain factor $C N$ can be approximated as

$$
C N \simeq\left(\frac{c^{2}}{1024 \eta_{e}^{4}}\right)^{1 / 6} \frac{\omega L I_{b}^{1 / 3}}{2 \pi V_{b}} F(\gamma a)
$$

The trends observed in the small-signal gain versus frequency curves of Figs. 10-12 can be readily understood from (A.4). The small-signal analysis predicts a strong increase in gain with in- creasing frequency, which is consistent with the CHRISTINE simulations of Figs. 10(a)-12(a), where there is roughly a $10 \mathrm{~dB}$ increase in gain over a $400-\mathrm{MHz}$ frequency span. The analysis also predicts an inversely proportional dependence of the gain on beam voltage, which is reflected in Fig. 10(a), where a 5\% increase in voltage results in as much as a $5 \mathrm{~dB}$ decrease in small-signal gain. The impact of voltage variations on the relative phase shift, as seen in Fig. 10(b), can be understood as the ability of the beam voltage to match the electron velocities to the phase velocity of the propagating electromagnetic wave on the helix - the closer the match, the more electron kinetic energy will be given up to the wave, slowing the beam and increasing the phase delay through the helix.

On the other hand, the gain should be largely independent of beam radius, as the impedance reduction factor - the only factor dependent on $r_{b}$-is approximately unity for this analysis. As seen in Fig. 11, the code is consistent with small-signal theory; the gain varies only an approximately $\pm 0.5 \mathrm{~dB}$ for $\pm 5 \%$ variations in $r_{b}$, and the relative phase shift displays a similar insensitivity.

Equation (A.4) does not describe the contribution of the dielectric helix support rods. However, their effect can be understood qualitatively; dielectric support rods have the effect of capacitively loading the helix, reducing the impedance and phase velocity on the circuit; in general, higher effective dielectric constants will result in increased phase delay through the TWT. Similar to variations in beam voltage, variations in the effective dielectric constant affect the the beam-wave coupling efficiency, this time by altering the phase velocity of the propagating electromagnetic wave relative to the velocity of the beam electrons. As expected, the gain and phase sensitivities of the dielectric variation case, plotted in Fig. 12, are similar to the voltage variation case of Fig. 10.

\section{APPENDIX B \\ DeVelopment of A Simple MultifRequency Model}

To further evaluate the utility of the CHRISTINE multifrequency model, it is instructive to compare its results with those of a simpler, gain-invariant, multifrequency model. The simple multifrequency model is developed below; model predictions are compared with CHRISTINE and experiment in Section IV-B.

For single-frequency TWT operation, the normalized, timedependent RF input and output voltages can be written as

$$
\begin{aligned}
a_{\text {in }}(t) & =\hat{a}_{\text {in }} e^{-\imath \omega t}+\text { c.c. } \\
a_{\text {out }}(t) & =\hat{a}_{\text {out }} e^{-\imath \omega t}+\text { c.c. }
\end{aligned}
$$

where the normalized input amplitude $\hat{a}_{\text {in }}$ is related to the RF drive power by $P_{\text {in }}=\left|a_{\text {in }}\right|^{2}$, and the normalized output amplitude $\hat{a}_{\text {out }}$ is related to the TWT output power $P_{\text {out }}=\left|a_{\text {out }}\right|^{2}$.

The magnitude of the amplified output voltage is related to the magnitude of the input voltage by a complex transfer function

$$
\hat{a}_{\text {out }}=g\left(\left|a_{\text {in }}\right|^{2}\right) \hat{a}_{\text {in }}
$$

where $g$, the complex nonlinear gain, is a function of the RF drive power. The magnitude $|g|$ and phase $\phi$ of the complex 
gain function can be approximated by a polynomial fit to the empirically measured gain of the TWT

$$
\begin{aligned}
|g| & =g_{0}+g_{1}\left|a_{\text {in }}\right|^{2}+g_{2}\left|a_{\text {in }}\right|^{4}+\cdots \\
\phi & =\Phi_{0}+\Phi_{1}\left|a_{\text {in }}\right|^{2}+\Phi_{2}\left|a_{\text {in }}\right|^{2}+\cdots
\end{aligned}
$$

Equation (B.2) strictly applies to a single-frequency signal. However, it can also be applied to a multifrequency signal with a sufficiently narrow bandwidth centered at $\omega_{0}$. In this case, $\hat{a}_{\text {in }}$ and $\hat{a}_{\text {out }}$ are time-dependent, slowly varying envelopes. The signal bandwidth must be small enough so that the function $g$ does not vary appreciably over the bandwidth. For a discrete set of for frequency inputs, $\omega_{n}=\omega_{0}+n \Delta \omega$, (B.1a) can be rewritten as

$$
\begin{aligned}
a_{\text {in }}(t) & =e^{-\imath \omega_{\mathbf{0}} t}\left[\sum_{n} \hat{a}_{\mathbf{i n}, n} e^{-\imath n \Delta \omega t}\right]+\text { c.c. } \\
a_{\text {out }}(t) & =e^{-\imath \omega_{\mathbf{0}} t}\left[\sum_{n} \hat{a}_{\mathrm{out}, n} e^{-\imath n \Delta \omega t}\right]+\text { c.c. }
\end{aligned}
$$

Following (B.2), the output amplitudes corresponding to the $\omega_{n}$ frequency components are expressed by the complex gain transfer function, where $g$ is a polynomial derived from a fit to data a single frequency

$$
\begin{aligned}
\hat{a}_{\mathrm{out}, n} e^{-\imath n \Delta \omega t}= & g\left(\left|\sum_{n} \hat{a}_{\mathbf{i n}, n} e^{-\imath n \Delta \omega t}\right|^{2}\right) \\
& \cdot \sum_{n} \hat{a}_{\mathbf{i n}, n} e^{-i n \Delta \omega t}
\end{aligned}
$$

and the normalized output voltage amplitude corresponding to the $m$ th frequency component is given by

$$
\begin{aligned}
\hat{a}_{\text {out }, m}= & \int \frac{d t \Delta \omega}{2 \pi} e^{\imath m \Delta \omega t} \times g\left(\left|\sum_{n} \hat{a}_{\mathbf{i n}, n} e^{-\imath n \Delta \omega t}\right|^{2}\right) \\
& \cdot \sum_{n} \hat{a}_{\mathbf{i n}, n} e^{-\imath n \Delta \omega t} .
\end{aligned}
$$

The RF power in the $m$ th frequency component, originating from the nonlinear beating of multiple tones, intermodulation, etc., is obtained from (B.6),

$$
P_{\text {out }, m}=\left|\hat{a}_{\text {out }, m}\right|^{2} \text {. }
$$

\section{ACKNOWLEDGMENT}

The authors would like to acknowledge the generous contributions of the Hughes Electron Dynamics Division for their loan of the $8537 \mathrm{H}$ TWTA and for providing corresponding design and fabrication data. In particular, the authors wish to thank Dr. A. Adler and Dr. W. Menninger of Hughes EDD, Dr. D. Gregoire of HRL Laboratories, and Dr. B. Danly and J. McDonald of the Naval Research Laboratory for their time and expertise.

\section{REFERENCES}

[1] T. M. Antonsen Jr. and B. Levush, "CHRISTINE: A multifrequency parametric simulation code for traveling-wave tube amplifiers,", NRL Rep. 97-9845, 1997.

[2] D. Chernin, T. M. Antonsen Jr., and B. Levush, "Exact treatment of the dispersion and beam interaction impedance of a thin tape helix surrounded by a radially stratified dielectric," IEEE Trans. Electron Dev., vol. 46, no. 7, pp. 1472-1483, July 1999.

[3] T. M. Antonsen Jr. and B. Levush, "Traveling-wave tube devices with nonlinear dielectric elements," IEEE Trans. Plasma Sci., vol. 26, no. 3, pp. 774-786, 1998.

[4] P. B. Kenington, "Methods linearize rf transmitters and power amps," Microwaves and RF, (Part 1), pp. 102-116, Dec. 1998. Jan. 1999, pp. 79-89 (Part 2).

[5] J. D. Wilson, "A simulated annealing algorithm for optimizing rf power efficiency in coupled-cavity traveling-wave tubes," IEEE Trans. Electron Dev., vol. 44, no. 12, pp. 2295-2299, Dec. 1997.

[6] J. E. Rowe, Nonlinear Electron-Wave Interaction Phenomena. New York: Academic, 1965.

[7] M. K. Scherba and J. E. Rowe, "Characteristics of multi-signal and noise-modulated high-power microwave amplifiers," IEEE Trans. Electron Dev., vol. ED-18, no. 1, pp. 11-34, Jan. 1971.

[8] H. K. Detweiler, "Characteristics of magnetically focused large signal traveling-wave amplifiers," Ph.D. dissertation, Univ. of Michigan, 1968.

[9] N. J. Dionne, "Harmonic generation in octave bandwidth traveling-wave tubes," IEEE Trans. Electron Dev., vol. ED-4, p. 365, 1970.

[10] A. J. Giarola, "A theoretical description for the multiple-signal operation of a TWT," IEEE Trans. Electron Dev., vol. ED-15, p. 381, 1968.

[11] D. R. Whaley, C. M. Armstrong, B. Gannon, G. Groshart, E. Hurt, J. Hutchins, M. Roscoe, T. M. Antonsen Jr., and B. Levush, "Sixty-percentefficient miniature C-band vacuum power booster for the microwave power module," IEEE Trans. Plasma Sci., vol. 26, no. 3, pp. 912-921, 1998.

[12] M. A. Basten, D. R. Whaley, and C. M. Armstrong, "Advanced modeling of helix TWTs," presented at the Conf. Record-1998 IEEE Int. Conf. Plasma Sci., Raleigh, NC, June 1-4, 1998.

[13] W. L. Menninger, private communication.

[14] D. J. Gregoire, private communication.

[15] O. Shimbo, "Effects of intermodulation, AM-PM conversion, and additive noise in multicarrier TWT systems," in Proc. IEEE, vol. 59, Feb. 1971, pp. 230-238.

[16] — , Transmission Analysis in Communications Systems. Rockville, Maryland: Computer Science Press, 1988, vol. 2.

[17] J. R. Pierce, Traveling Wave Tubes. Princeton, NJ: Nostrand, 1950.

David K. Abe (M'92) received the B.S. (engineering) degree with honors from Harvey Mudd College, Claremont, CA, in 1981, the M.S.E.E. degree from the University of California, Davis, in 1988, and the Ph.D. degree in electrophysics from the University of Maryland, College Park, in 1992. From 1982 to 1988, he worked at the Lawrence Livermore National Laboratory, Livermore, CA, as an Electronics Engineer, supporting the nuclear design program. From 1992 to 1994, he was employed as a Research Scientist with Berkeley Research Associates, Springfield, VA, where he worked on pulsed power and high power microwave projects. He joined the Army Research Laboratory in 1994, where he pursued projects in electromagnetic effects and high power microwave generation. In 1997, he joined the Vacuum Electronics Branch of the Naval Research Laboratory, Washington, DC, where he is currently pursuing research projects in the areas of linear beam, slow-wave microwave devices and the electronic properties of materials.

Dr. Abe is a Member of the American Physical Society and the Association of Old Crows.

Mai T. Ngô was born in Saigon, Vietnam, in June 1964. She received the B.S. and M.S. degrees in electrical engineering in 1987 and 1989, respectively, from Old Dominion University, Norfolk, VA.

Since 1989, she has been with Mission Research Corporation, Washington, DC. Since 1996, she has been an On-Site Contractor for the Naval Research Laboratory, Washington, DC, where she is actively involved in developing various microwaves sources, characterizing phase noise, and integrating a 94-GHz radar system. Her research interests include electron beam diagnostics, electro-optic sensors, high-power microwaves, and pulsed power.

Ms. Ngo is a member of Tau Beta Pi and Eta Kappa Nu. 
Baruch Levush (M'88-SM'90) received the M.Sc. degree in physics from Latvian University, Riga, Latvia, in 1972 and the Ph.D. degree in physics from Tel-Aviv University, Tel-Aviv, Israel, in 1981.

He received the Dr. Ch. Weizman Postdoctoral Fellowship and stayed for two years at the University of Maryland, College Park. From 1984 to 1985, he was a Research Scientist with Rafael Research Laboratory, Israel. In 1985, he joined the University of Maryland, where his research was focused on the physics of coherent radiation sources and the design of high-power microwave sources, such as gyrotrons, TWT's, BWO's, and free electron lasers. In 1993, he became a Senior Research Scientist at the Institute for Plasma Physics, University of Maryland. In 1995, he joined the Naval Research Laboratory, Washington, DC, as the Head of the Theory and Design Section of the Vacuum Electronics Branch. He is actively involved in developing theoretical models and computational tools for analyzing the operation of existing microwave vacuum devices and in inventing new concepts for high-power, high-frequency coherent radiation sources. He is the author and coauthor of more than 100 journal articles.

Dr. Levush is a Member of the American Physical Society.

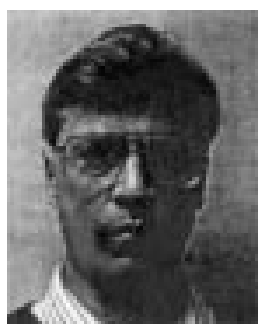

Thomas M. Antonsen, Jr. (M'87) was born in Hackensack, NJ in 1950. He received the Bachelor's degree in electrical engineering in 1973, and his Master's and Ph.D. degrees in 1976 and 1977, all from Cornell University. He was a National Research Council Post Doctoral Fellow at the Naval Research Laboratory in 1976-1977, and a Research Scientist in the Research Laboratory of Electronics at MIT from 1977 to 1980. In 1980, he moved to the University of Maryland where he joined the faculty of the departments of Electrical Engineering and Physics in 1984. He is currently a Professor of Physics and Electrical Engineering. He has held visiting appointments at the Institute for Theoretical Physics (U.C.S.B.), the Ecole Polytechnique Federale de Lausanne, Switzerland, and the Institute de Physique Theorique, Ecole Polytechnique, Palaiseau, France. He was selected as a Fellow of the Division of Plasma Physics of the American Physical Society in 1986.

Prof. Antonsen's research interests include the theory of magnetically confined plasmas, the theory and design of high power sources of coherent radiation, nonlinear dynamics in fluids, and the theory of the interaction of intense laser pulses and plasmas. He is the author and coauthor of more than 180 journal articles and coauthor of the book Principles of Free-electron Lasers. He has served on the editorial board of Physical Review Letters. The Physics of Fluids, and Comments on Plasma Physics.

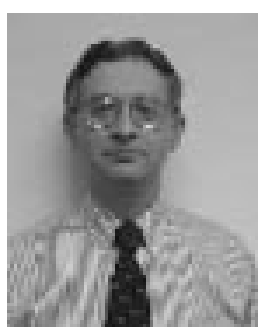

David P. Chernin received the A.B. and Ph.D. degrees in 1971 and 1976, respectively, in applied mathematics from Harvard University.

From 1976 to 1978, he was a Member of the Institute for Advanced Study, in Princeton, NJ, where he worked on problems in magnetic confinement fusion Since 1984, he has been at Science Applications International Corporation, McLean, VA, where he has contributed to multiple research efforts in the theory and simulation of beam-wave interactions in particle accelerators and microwave tubes.

Dr. Chernin presently serves as the Co-Director of the Center for Electromagnetic Science and Engineering at SAIC. He is a Member of the American Physical Society and the Society for Industrial and Applied Mathematics. 